\title{
Should wrong extraction site be classed as a never event?
}

\author{
V. Sampson ${ }^{1}$
}

Key points

Defines wrong site surgery.

Asks how the NHS is attempting to solve the issue of

the increase in wrong site surgeries.

Discusses flaws with the definition of a never event.

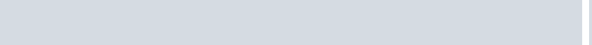

NHS England has set out plans for combatting the rise in never events in the realm of dentistry. This Opinion article investigates the root cause of never events, whether a wrong site surgery should be deemed a never event, and whether the approaches adopted to reduce risk exposure, such as local safety standards for invasive procedures (LocSSIPS), are helping or hindering safe medical care.

The term 'never event' was first introduced by Ken Kizer in 2001, in reference to shocking medical errors that should never have occurred. ${ }^{1}$ A never event is defined by NHS England as a potentially harmful incident that could have been avoided had the healthcare provider followed national guidelines. ${ }^{2}$ Wrong site surgery (WSS) is the most common medical error performed in the realm of dentistry. Twenty-five percent of all WSS are dental in nature, ${ }^{3}$ whereby a permanent tooth not intended to be extracted during that session is removed. Between April 2016 and March 2017, 44 incorrect teeth were extracted in England under NHS funded care, ${ }^{2}$ nine more than in the previous year. ${ }^{4}$ The question is, should wrong tooth extractions be deemed just as 'harmful' as or 'dangerous' as potentially life threatening never events such as maternal death from post-partum haemorrhage, or surgery on the wrong side of the brain? Furthermore, if the operating dental surgeon appears to have carried out all recommended safety precautions as dictated by the NHS guidelines and yet the incident still occurs, should the incident be categorised as a never event? Lastly, has the increase in WSS been effectively addressed by the NHS with the introduction of never events, or has the NHS created a culture of blame?

'Barts and the London School of Medicine and Dentistry Correspondence to: Victoria Sampson Email: victoriasampson@hotmail.com

Refereed Paper.

Accepted 14 May 2018

Published online 17 August 2018

DOI: $10.1038 /$ sj.bdj.2018.649
Many argue that if all safety precautions are performed and the incident still occurs, it cannot be categorised as a never event. As Professor Toft states during an external report of root cause analysis, most incorrect tooth extractions are caused by human error. ${ }^{5}$ The human error in this case is usually a 'perceptual error' which causes the operator to lose 'situational awareness'. In short, just like all humans, operators can be subject to confirmation bias, where they convince themselves they are extracting the tooth they believe they should be extracting. This means that no matter how many guidelines the NHS imposes in the hope of avoiding never events, sometimes they happen irrespective of guidance, due to human error. This is supported by the fact that even after the introduction of surgical safety checklists and procedures, there has still been a steady increase in the number of never events since 2013. ${ }^{2}$ Therefore, if a provider takes every recommended step to prevent occurrence and an incident still occurs, this argues strongly that the incident was not preventable and therefore not a never event. ${ }^{6}$ Labelling mistakes such as these as never events only creates a culture of blame and instils fear in clinicians. This fear, contrary to the common belief that it may help avoid future mistakes, would actually increase the likelihood of a mistake. Studies such as J. Yeow's in 2014 have shown a positive correlation between human error and stress. Stress is proven to divert one's attention and cause one to be in a less composed state of mind, and therefore more likely to make a mistake.
Reason's Swiss Cheese Model of accident causation is used in healthcare to analyse patient safety incidents, such as WSS. The paradigm explains that hazards are prevented by a series of barriers (in the case of the metaphor, each slice of cheese). Each barrier has a weakness (or a hole). If there is a trajectory of errors/weaknesses, a hazard will occur. ${ }^{7}$ This paradigm explains that a never event is not usually caused by a single error, but by a combination of factors and weaknesses both in the practitioner and the trust, resulting in a patient safety incident. Therefore, if the increase in WSS is to be addressed, a combination of weaknesses must be considered. Labelling a WSS a never event and effectively blaming a single clinician involved is therefore unjust, as mistakes happen when there is a combination of weaknesses and errors from the hospital, trust, clinician, and those working alongside them.

A similar blame culture used to exist in the aviation industry, prior to the tragic United Airlines Flight 173 crash in 1978. Due to dangerously low fuel, the pilot was forced to crash land mid-flight, causing the death of ten people. Following the crash, the pilot was involved in a seven year long court case, lost his pilot license, and came close to suicide on multiple occasions. There is a silver lining however; the aviation industry learnt from this crash that firstly; a blame culture is unjust, as in this case anyone could have noticed the low fuel levels, and secondly, that under pressure, 'human error' is inevitable and impossible to avoid. The issue was not that this particular pilot had made a mistake that no one else would have 
done, but that anyone in a similar situation, especially under stress, could have made the same mistake. Furthermore, they realised the importance of teamwork and communication, as it is every team member's responsibility to ensure passenger safety, not just the pilot's. As a result, the aviation industry transformed their training programme to ensure that pilots discuss and work alongside engineers and attendants to allow effective communication and team work. The outcome was dramatic, the number of deaths halved over 30 years (despite the nine-fold increase in air travel), thus showing how diversion from a blame culture is beneficial.

The NHS states that even if a tooth that is treatment planned to be extracted at a future appointment is inadvertently removed, it is still classified as a never event. Whilst there is general consensus that the inadvertent removal of a tooth is a deviation from the consent gained for that particular treatment, it is also questionable why such an event would be classed as a never event if it has caused no harm to the patient. The NHS guidelines specifically states that a never event's defining factor is that the event has or will cause permanent damage to the patient. In this situation, no harm has occurred and the dentist can then extract the tooth initially planned to be extracted, at the next appointment. Whilst NHS trusts should investigate any incident, the penalties associated with never events can be punitive and involve significant financial penalty. As a result, the NHS has created a stigma instead of promoting learning from one's mistakes. ${ }^{8}$ In situations as highlighted above, should they be deemed just as grave as an extraction of a virgin tooth and receive the same scrutiny and punishment?

Lastly, the NHS has defined a WSS in terms of dentistry as an extraction of a permanent tooth not intended to be extracted. This implies that the incorrect extraction of a deciduous tooth is not a never event, even though sometimes the consequences of incorrect extraction of a deciduous tooth can meet the 'serious harm' threshold. An example would be if the successor of the wrongfully extracted primary tooth is developmentally absent. This would result in long-term corrective treatment for the patient and therefore is perhaps more 'serious' than, for example, removing an incorrect permanent tooth which is treatment planned to be removed at a later appointment.

On the other hand, tooth loss has a direct correlation with reduction in oral health-related quality of life. ${ }^{9}$ Commonly, patients will report impairment of mastication and aesthetics and will often opt for costly dental treatments to replace edentulous areas. If the incorrect tooth is extracted, not only has the patient lost a vital, healthy tooth, but they will also eventually have to lose the tooth primarily proposed to be extracted. As Hippocrates famously said, 'first do no harm. Dentists have a duty of care to their patients, as outlined by the GDC, to 'put patients' interests first' and therefore provide treatment in their best interest..$^{10}$ Never events highlight weak areas within an organisation's safety processes and patient safety. Therefore, they are integral for the improvement and development of the NHS system. If WSSs were not reported and not granted the gravity they deserve, the NHS would be at a risk of reduced patient safety, and would not learn from its mistakes. ${ }^{11}$

In conclusion, the idea of reporting events that put a patient at harm and learning from these events is undoubtedly a great advancement for the NHS. It is fundamental that clinicians continue to do what they can to make patient care possible and to reduce any exposure to risk. However, the term never event is an umbrella term for a myriad of events, across a wide spectrum. It is unfounded to put a WSS of a tooth that was planned to be extracted at a future appointment at the same level as, for example, a surgery on the wrong side of the brain. If the defining factors of a never event are that they cause irreversible harm to the patient, then extracting a tooth that is meant to ultimately be extracted cannot be deemed a never event. Furthermore, another defining factor of a never event is that the event has occurred due to the clinician not following the national guidelines on safety precautions. As already stressed, many WSSs happen irrespective of the clinician following the guidelines, and have actually increased regardless of introduction of more safety precautions. This makes one question whether the approaches that have been adopted to reduce risk exposure, such as introducing LocSSIPS, ${ }^{12}$ are helping or hindering safe medical care. The MDU strongly argues that the term never event is a misnomer and shines the spotlight on a select number of preventable events, eclipsing other serious, life threatening events that could also have been prevented. ${ }^{8}$ An example would be the administration of penicillin to a patient that suffers from a penicillin allergy. By creating a list of never events, not only is the NHS suggesting that other equally harmful events are not as serious as they have not made it onto the list, but it can also attach a stigma to these particular events and creates a culture of blame as opposed to a culture of learning and openness. ${ }^{13} \mathrm{~A}$ parallel can be drawn between the aviation industry and the NHS. Whilst the human body and the NHS is arguably more complex and unpredictable than aviation, the idea that a blame culture is counterproductive and actually reduces patient or passenger safety is evident. Healthcare providers should always aim to reinforce patient safety. At any point during treatment, a mistake can happen and unfortunately, the NHS cannot create a guideline to avoid every mistake. Often, these mistakes come down to human error and whilst we should all learn from our mistakes and avoid making them, sometimes they are unavoidable and therefore should be seen as a learning curve as opposed to a disastrous event for the trust. Although WSS should never happen, they should not be labelled as a never event. Perhaps the NHS should take a leaf out of the aviation industry's book, and focus on prevention of never events by promoting team work, communication and managing workload, as opposed to creating a punitive blame culture.

1. Lembitz A, Clarke T J. Clarifying 'never events' and introducing 'always events'. Patient Saf Surg 2009; 3: 26. 2. NHS England. Provisional publication of Never Events reported as occurring between 1 April 2016 and 28 February 2017. https://improvement.nhs.uk/ documents/2347/Never_Events_1_April_2016_-_31_ March_2017_FINAL_V2.pdf (accessed August 2018).

3. Pemberton M N, Ashley M P, Saksena A, Dickson S. Wrong tooth extraction: an examination of 'Never Event' data. Br J Oral Maxillofac Surg 2017; 55: 187-188.

4. NHS Improvement. Never Events reported as occurring between 1 April 2015 and 31 March 2016 - final update. 2016. Available at https://improvement.nhs. uk/documents/674/NE_data_report_1_April_2015__31_March_2016_FINAL_V2.pdf (accessed July 2018).

5. Oxford University Hospitals. External Review of 'Never Events' that occurred at Oxford University Hospitals NHS Trust during the period 13 September 2013 to 26 March 2015. 2016. Available at https://www.ouh.nhs.uk/ about/trust-board/2016/january/documents/TB2016.07never-event-review.pdf (accessed July 2018).

6. Department of Health. The 'never events' list 2011/12. Policy framework for use in the NHS. 2012. Available at https://www.gov.uk/government/publications/the-never-events-list-for-2011-12 (accessed July 2018).

7. Perneger T. The Swiss cheese model of safety incidents: are there holes in the metaphor? BMC Health Serv Res 2005; 5: 71.

8. Tomkins C. Never Events- a misnomer. MDU 2013. Avail able at https://www.themdu.com/guidance-and-advice/ journals/mdu-journal-november-2013/never-events---amisnomer (accessed July 2018).

9. Gerritsen A E, Allen P F, Witter D J, Bronkhorst E M, Creugers N H. Tooth loss and oral health-related quality of life: a systematic review and meta-analysis. Health Qual Life Outcomes 2010; 8: 126.

10. General Dental Council. Standards for the Dental Team. Available at https://www.gdc-uk.org/api/files/NEW\%20 Standards\%20for\%20the\%20Dental\%20Team.pdf (accessed July 2018).

11. NHS Improvement. Revised Never Events Policy and Framework. Available at https://improvement.nhs.uk/resources/ never-events-policy-and-framework/ (accessed July 2018).

12. NHS England. Tool kit for 'Local Safety Standards for Invasive Procedures' (LocSSIPS) for wrong site extraction in Dentistry. 2015.

13. MDU. Never events should be learned from alongside all patient incidents. The MDU, 2016. Available at https:// www.themdu.com/about-mdu/our-impact/our-impact-archive/never-events-do-happen (accessed July 2018). 